\title{
Immunocytochemistry of CD146 is useful to discriminate between malignant pleural mesothelioma and reactive mesothelium
}

\author{
Ayuko Sato ${ }^{1}$, Ikuko Torii ${ }^{1}$, Yoshihiro Okamura ${ }^{1}$, Tadashi Yamamoto ${ }^{1}$, Takashi Nishigami ${ }^{1}$, \\ Tatsuki R Kataoka ${ }^{1}$, Misa Song ${ }^{1}$, Seiki Hasegawa ${ }^{2}$, Takashi Nakano ${ }^{3}$, Toshiaki Kamei ${ }^{4}$ \\ and Tohru Tsujimura ${ }^{1}$ \\ ${ }^{1}$ Department of Pathology, Hyogo College of Medicine, Hyogo, Japan; ${ }^{2}$ Department of General Thoracic \\ Surgery, Hyogo College of Medicine, Hyogo, Japan; ${ }^{3}$ Division of Respiratory Medicine, Department of Internal \\ Medicine, Hyogo College of Medicine, Hyogo, Japan and ${ }^{4}$ Division of Pathology, Yamaguchi Grand Medical \\ Center, Yamaguchi, Japan
}

\begin{abstract}
Malignant pleural mesothelioma is a refractory tumor with poor prognosis associated with asbestos exposure. Pleural effusion is frequently observed in patients with malignant pleural mesothelioma, and cytological analysis is effective to detect malignant pleural mesothelioma. However, cytological discrimination between malignant pleural mesothelioma and reactive mesothelium is often difficult. Increased expression of CD146, a cell adhesion molecule, has been reported to be closely associated with an advanced stage of malignant melanoma, prostate cancer, and ovarian cancer. In this study, to evaluate the diagnostic utility of CD146 for discrimination between malignant pleural mesothelioma and reactive mesothelium, we examined immunocytochemical expression of CD146 in malignant pleural mesothelioma and reactive mesothelium using two clones of CD146 antibody, OJ79 and EPR3208, on smear specimens of effusion fluids. Immunocytochemical stains were semiquantitatively scored on the basis of immunostaining intensity ( 0 , negative; 1 , weak positive; 2 , moderate positive; and 3, strong positive). CD146 expression was detected in 15 of 16 malignant pleural mesothelioma with median immunostaining score of 3 by OJ79, and in 19 of 21 malignant pleural mesothelioma with median immunostaining score of 2 by EPR3208. Strong immunoreactivity of CD146 was observed at the apposing surfaces of cell-cell interactions on the plasma membrane of mesothelioma cells. In addition, one OJ79-negative case of malignant pleural mesothelioma was positive for CD146 by EPR3208 and two EPR3208negative cases of malignant pleural mesothelioma were CD146 positive by OJ79, showing that all 23 malignant pleural mesothelioma cases were positive for CD146 by either OJ79 or EPR3208. On the other hand, CD146 expression was undetectable in all reactive mesothelium cases by OJ79 and EPR3208. The sensitivity of OJ79 and EPR3208 was 94 and $90 \%$, respectively, and the specificity was $100 \%$ for both clones. We propose that CD146 is a sensitive and specific immunocytochemical marker enabling differential diagnosis of malignant pleural mesothelioma from reactive mesothelium.

Modern Pathology (2010) 23, 1458-1466; doi:10.1038/modpathol.2010.134; published online 23 July 2010
\end{abstract}

Keywords: CD146; cytology; immunocytochemistry; malignant mesothelioma; pleural effusion; reactive mesothelium

Malignant pleural mesothelioma is an aggressive tumor associated with asbestos exposure. Malignant pleural mesothelioma was once a rare disease, but

Correspondence: Dr T Tsujimura, MD, PhD, Department of Pathology, Hyogo College of Medicine, 1-1 Mukogawa-cho, Nishinomiya, Hyogo 663-8501, Japan.

E-mail: tohru@hyo-med.ac.jp

Received 30 April 2010; revised 10 June 2010; accepted 11 June 2010; published online 23 July 2010 its incidence is increasing worldwide and expected to peak in around 2020. ${ }^{1}$ Malignant pleural mesothelioma is characterized by high resistance to conventional therapies and poor prognosis, with the median survival in the range of 9-17 months after diagnosis. ${ }^{2}$ The poor prognosis is, at least in part, because of the difficulty of precise diagnosis until the disease progresses to an advanced stage. The initial clinical presentation for patients with malignant pleural mesothelioma is frequently 
dyspnea and/or chest pain due to large pleural effusions. ${ }^{3}$ Therefore, cytological analysis of pleural effusions is particularly important for patients with a history of asbestos exposure in the absence of other major clinical features.

The pathological diagnosis of malignant pleural mesothelioma in effusion cytology is based on the demonstration of mesothelial nature and malignancy of cells. Cytopathological features of mesothelioma are characterized by mesothelial cells with nuclear atypia, multinucleation, cell-in-cell engulfment, and mirror ball-like cell cluster formation, which are useful in discrimination from other diseases. $^{4-7}$ Effusion cytology for differential diagnosis of malignant pleural mesothelioma and adenocarcinoma uses immunostaining panels with mesothelial markers, such as calretinin, podoplanin (D2-40), Wilms' tumor 1 protein, and cytokeratin $5 / 6$, and carcinoma markers, such as epithelialrelated antigen (MOC-31) and carcinoembryonic antigen. ${ }^{8,9}$ On the other hand, distinction of malignant pleural mesothelioma from reactive mesothelium is challenging, because of the similarity of morphology and the lack of reliable discriminating markers in effusion cytology. Several markers, including epithelial membrane antigen (EMA), desmin, glucose transporter-1 (GLUT-1), and p53, have been reported to be of use in distinguishing malignant pleural mesothelioma and reactive mesothelium in effusion cytology. However, the results of analysis using these markers vary greatly among reports. ${ }^{10-18}$

Cell adhesion molecules, involved in homophilic and heterophilic cell-cell interactions, have important roles in organogenesis and maintenance of tissue structure and function. ${ }^{19}$ Aberrant expression of cell adhesion molecules is frequently observed during tumor development and progression, probably because of increased cell motility, allowing tumor invasion and metastasis. ${ }^{20} \mathrm{CD} 146$, also known as melanoma cell adhesion molecule or MUC18, is a transmembrane glycoprotein belonging to the immunoglobulin superfamily that functions as a $\mathrm{Ca}^{2+}$ independent adhesion molecule. ${ }^{21}$ Increased CD146 expression has been shown to be closely associated with an advanced stage of malignant melanoma, prostate cancer, and ovarian cancer. ${ }^{21-25}$ However, the relation between CD146 expression and the malignancy of mesothelioma has not been investigated. The purpose of the present study was to evaluate the utility of CD146 detection for distinguishing malignant pleural mesothelioma and reactive mesothelium in diagnostic cytology.

\section{Materials and methods}

\section{Cases}

A total of 51 cases, 23 malignant pleural mesothelioma and 28 reactive mesothelium, were included in this study (Table 1). A total of 47 cytological smears for immunocytochemistry were prepared from pleural, peritoneal, or pericardial effusions and pleural washing fluids at the Hospital of Hyogo College of Medicine. The diagnosis of malignant pleural mesothelioma was confirmed by histopathological examination of pleural biopsy or resection tissues. The remaining four cytological smears for immunocytochemistry were prepared from Papanicolaou-stained smear slides by cell transfer technique after cytological analysis at the Yamaguchi Grand Medical Center. Briefly, the cytological materials of Papanicolaou-stained smear slides were completely covered with Malinol (Muto Pure Chemicals, Tokyo, Japan) after removing cover glass. The layer of Malinol with smeared cells was removed from each slide, and the layer was divided into several pieces. The piece with smeared cells was transferred onto a new slide. Smears reproduced on the slides were immunostained with antibodies.

This work has been carried out in accordance with the Declaration of Helsinki (2000) of the World Medical Association and is approved by institutional review board.

\section{Immunocytochemistry}

Cytological smears were fixed with alcohol. Two clones of anti-CD146 antibody, OJ79 of mouse monoclonal antibody (1:200 dilution; Invitrogen, Carlsbad, CA, USA) and EPR3208 of rabbit monoclonal antibody (1:200 dilution; Epitomics, Burlingame, CA, USA), and anti-EMA mouse monoclonal antibody (1:40 dilution, clone E29; Dako Cytomation, Glostrup, Denmark) were used as primary antibodies. For EPR3208, cytological smears were heated in Target Retrieval Solution (S1700; Dako Cytomation) at $98^{\circ} \mathrm{C}$ for $20 \mathrm{~min}$ to facilitate antigen retrieval. Cytological smears were incubated with each primary antibody, and then with an anti-mouse or rabbit immunoglobulin antibody using ChemMate EnVision Kit (Dako Cytomation). Immunoreacted cells were visualized with 3,3'-diaminobenzidine, and nuclei were lightly counterstained with hematoxylin. Immunocytochemical stainings were semiquantitatively scored on the basis of immunostaining intensity (0, negative; 1 , weak positive; 2 , moderate positive, and 3 , strong positive). 'Positive' indicates the presence of cells with intensity 1 or higher signals in specimens. 'Negative' indicates the presence of cells with no or faint signals in specimens.

\section{Statistical Analysis}

Immunostaining scores were compared between malignant pleural mesothelioma and reactive mesothelium by Mann-Whitney $U$-nonparametric test. Two-tailed $P$-values below 0.05 were considered as statistically significant. 
Table 1 Clinical characteristics and immunocytochemical findings

\begin{tabular}{|c|c|c|c|c|c|c|c|}
\hline Case no. & Age & Sex & Disease & Source & Class & $E M A$ & $C D 146$ \\
\hline
\end{tabular}

\begin{tabular}{cccc}
\hline \multicolumn{4}{c}{ Malignant pleural mesothelioma } \\
1 & 73 & M & \\
2 & 65 & MPM \\
3 & 48 & M & MPM \\
4 & 65 & M & MPM \\
5 & 44 & M & MPM \\
6 & 73 & M & MPM \\
7 & 71 & M & MPM \\
8 & 67 & M & MPM \\
9 & 57 & M & MPM \\
10 & 69 & F & MPM \\
11 & 65 & M & MPM \\
12 & 74 & M & MPM \\
13 & 51 & M & MPM \\
14 & 63 & M & MPM \\
15 & 68 & M & MPM \\
16 & 63 & M & MPM \\
17 & 53 & M & MPM \\
18 & 61 & M & MPM \\
19 & 75 & M & MPM \\
20 & 68 & M & MPM \\
21 & 92 & M & MPM \\
22 & 76 & M & MPM \\
23 & 69 & M & MPM
\end{tabular}

Reactive mesothelium

\begin{tabular}{|c|c|c|c|c|c|c|c|c|}
\hline 1 & 53 & $\mathrm{~F}$ & Lung cancer & $\mathrm{Pl}$ & $\mathrm{V}^{\mathrm{d}}$ & - & - & NP \\
\hline 2 & 82 & $\mathrm{M}$ & Lung cancer & $\mathrm{Pl}$ & II & - & - & - \\
\hline 3 & 69 & $\mathrm{~F}$ & Lung cancer & $\mathrm{Pl}$ & II & - & - & - \\
\hline 4 & 56 & $\mathrm{M}$ & Lung cancer & $\mathrm{Pl}$ & II & - & NP & - \\
\hline 5 & 63 & M & Lung cancer & PW & II & NP & - & NP \\
\hline 6 & 59 & $\mathrm{M}$ & Lung cancer & PW & II & - & - & - \\
\hline 7 & 61 & M & HCC & $\mathrm{Pl}$ & II & - $^{\mathrm{e}}$ & NP & - \\
\hline 8 & 54 & $\mathrm{M}$ & HCC & $\mathrm{Pl}$ & II & $1+$ & NP & - \\
\hline 9 & 77 & $\mathrm{~F}$ & HCC & $\mathrm{Pl}$ & II & - & NP & - \\
\hline 10 & 75 & $\mathrm{~F}$ & Breast cancer & $\mathrm{Pl}$ & $I V^{d}$ & - & - & - \\
\hline 11 & 44 & $\mathrm{~F}$ & Cervical cancer & $\mathrm{Pl}$ & II & $-^{\mathrm{e}}$ & NP & - \\
\hline 12 & 60 & $\mathrm{M}$ & Gastric cancer & $\mathrm{Pl}$ & II & - & - & NP \\
\hline 13 & 71 & $\mathrm{M}$ & Pneumonia & $\mathrm{Pl}$ & III & $1+$ & - & NP \\
\hline 14 & 65 & $\mathrm{M}$ & Pneumonia & $\mathrm{Pl}$ & II & - & NP & - \\
\hline 15 & 58 & $\mathrm{M}$ & Pneumothorax & $\mathrm{Pl}$ & II & - & - & NP \\
\hline 16 & 87 & $\mathrm{M}$ & Hemothorax & $\mathrm{Pl}$ & II & NP & - & NP \\
\hline 17 & 84 & $\mathrm{~F}$ & Pleuritis & $\mathrm{Pl}$ & II & $1+$ & - & NP \\
\hline 18 & 85 & $\mathrm{M}$ & Pleuritis & $\mathrm{Pl}$ & II & - $^{\mathrm{e}}$ & NP & - \\
\hline 19 & 78 & $\mathrm{M}$ & Heart failure & Pc & II & - & NP & - \\
\hline 20 & 90 & $\mathrm{M}$ & Heart failure & $\mathrm{Pl}$ & II & - & NP & - \\
\hline 21 & 91 & $\mathrm{~F}$ & Heart failure & $\mathrm{Pl}$ & II & - $^{\mathrm{e}}$ & NP & - \\
\hline 22 & 72 & $\mathrm{M}$ & Chronic renal failure & $\mathrm{Pl}$ & II & $-^{\mathrm{e}}$ & NP & - \\
\hline 23 & 69 & $\mathrm{M}$ & Chronic renal failure & $\mathrm{Pl}$ & II & - & NP & - \\
\hline 24 & 48 & $\mathrm{M}$ & Pleural effusion ${ }^{\mathrm{f}}$ & $\mathrm{Pl}$ & II & - & NP & - \\
\hline 25 & 96 & $\mathrm{~F}$ & Pleural effusion ${ }^{\mathrm{f}}$ & $\mathrm{Pl}$ & II & - & NP & - \\
\hline 26 & 74 & $\mathrm{~F}$ & Pleural effusion ${ }^{\mathrm{f}}$ & $\mathrm{Pl}$ & II & - & NP & - \\
\hline 27 & 79 & $\mathrm{M}$ & Pleural effusion ${ }^{\mathrm{f}}$ & $\mathrm{Pl}$ & II & - & NP & - \\
\hline 28 & 76 & $\mathrm{M}$ & Pleural effusion ${ }^{\mathrm{f}}$ & $\mathrm{Pl}$ & II & - & NP & - \\
\hline
\end{tabular}

Abbreviations: As, ascitic fluid; F, female; HCC, hepatocellular carcinoma; M, male; MPM, malignant pleural mesothelioma; NP, not performed; Pc, pericardial effusion; Pl, pleural effusion; PW, pleural washing fluids.

${ }^{a}$ Biphasic type. Other cases were histopathologically diagnosed as epithelioid type.

${ }^{\mathrm{b}}$ Specimens were prepared by cell transfer technique.

${ }^{\mathrm{c}}$ Ascitic fluids were used for immunocytochemistry with OJ79 and pericardial effusion for immunocytochemistry with EPR3208.

${ }^{\mathrm{d}}$ Cytology class (class V or IV) for the primary disease (lung cancer or breast cancer).

${ }^{\mathrm{e}}$ Almost all reactive mesothelial cells were negative for EMA, but some reactive mesothelial cells were positive.

${ }^{\mathrm{f}}$ Pleural effusion of uncertain cause. 


\section{Results}

\section{Patients' Characteristics}

Clinical characteristics of 23 malignant pleural mesothelioma and 28 reactive mesothelium cases are shown in Table 1. Malignant pleural mesothelioma, one of the biphasic type (case no. 1) and 22 of the epithelioid type (case nos. 2-23), were diagnosed based on histopathological examinations of pleural biopsy or resection tissues. Patients with malignant pleural mesothelioma comprised 22 men and 1 woman aged 44-92 years with the median age of 67 years. Patients with pleural effusion, pericardial effusion, or pleural washing fluids, including reactive mesothelial cells, comprised 19 men and 9 women aged 44-96 years with the median age of 72 years.

\section{CD146 expression in malignant pleural mesothelioma and reactive mesothelium}

We examined CD146 expression in malignant pleural mesothelioma and reactive mesothelium by immunocytochemical staining of cytological smears using two clones of anti-CD146 monoclonal antibodies, OJ79 and EPR3208. Results are summarized in Table 1.

The CD146 expression was detected in 15 of 16 (94\%) specimens from malignant pleural mesothelioma cases using OJ79. Most of them showed strong expression on the mesothelioma cell membrane, with positive signals frequently observed at the apposing surfaces of cell-cell interactions (Figures 1a, 1b, 3a and 3c). In some specimens, immunostaining intensity was variable among mesothelioma cells (intensity 1-3). In contrast, CD146 expression was undetectable by OJ79 in all (11 of 11) reactive mesothelium cases (Figure 1c and d).

To confirm CD146 expression in malignant pleural mesothelioma, we repeated analysis of CD146 expression using another anti-CD146 rabbit monoclonal antibody, clone EPR3208. CD146 expression was detected in 19 of 21 (90\%) malignant pleural mesothelioma (Figures 2a, 2b, 3a and 3d). EPR3208 was almost equal to OJ79 in detectability for mesothelioma cells, but signal strength with EPR3208 is a little weaker than that with OJ79. In three cases, OJ79 and EPR3208 gave inconsistent results, with two cases (malignant pleural mesothelioma-case nos. 2 and 7) being positive for OJ79 and negative for EPR3208 and one case (malignant pleural mesothelioma-case no.15) being positive for EPR3208 and negative for OJ79. However, these findings show that all 23 malignant pleural mesothelioma cases were positive for CD146 by either OJ79 or EPR3208. On the other hand, CD146 expression was undetectable by EPR3208 in all (21 of 21) reactive mesothelium cases (Figure 2c and d).

Immunocytochemical staining for EMA was performed in 20 of 23 malignant pleural mesothelioma cases and in 26 of 28 reactive mesothelium cases used for CD146 evaluations. EMA expression was detected in 19 of 20 (95\%) malignant pleural mesothelioma, but one EMA-negative malignant pleural mesothelioma case showed CD146-positive staining (malignant pleural mesothelioma-case no.12). In reactive mesothelium, 23 of $26(88 \%)$ cases were negative for both EMA and CD146 staining, and the remaining 3 cases of reactive mesothelium were EMA positive and CD146 negative (reactive mesothelium-case nos. 8, 13, and 17). Both EMA and CD146 were principally expressed on the plasma membrane of mesothelioma cells. However, EMA expression was mostly observed at the periphery of cell clusters (Figure 3a and b), whereas CD146 at the apposing surfaces of cell-cell interactions (Figure 3a, c and d).

Immunostaining scores with OJ79 and EPR3208 are graphically shown in Figure 4. OJ79 showed a median score 3 for malignant pleural mesothelioma and 0 for reactive mesothelium $(P<0.001)$, and EPR3208 showed a median score 2 for malignant pleural mesothelioma and 0 for reactive mesothelium $(P<0.001)$. When the level of $\geqslant 1$ was considered to be positive, the sensitivity and specificity of OJ79 were 94 and $100 \%$ and those of EPR3208 were 90 and $100 \%$, respectively. These results indicate the usefulness of CD146 in discrimination between malignant pleural mesothelioma and reactive mesothelium.

\section{Discussion}

The cytomorphological scoring system for malignant mesothelioma in effusion cytology has been introduced for differential diagnosis of malignant mesothelioma and reactive mesothelium. ${ }^{26}$ However, to achieve the differential diagnosis successfully, numerous cytomorphological parameters are required. A variety of markers, such as EMA, desmin, GLUT-1, and p53, have been reported to be useful in the discrimination of malignant mesothelioma and reactive mesothelium, but the results are not consistent. ${ }^{10-16}$ For instance, EMA is considered to be the most reliable marker for malignant mesothelioma, detecting more than $70 \%$ of malignant mesothelioma cases, but it is also found in $0-70 \%$ of reactive mesothelium cases. ${ }^{16-18}$ In this study, we explored the possibility of using CD146 as a marker for distinguishing between malignant pleural mesothelioma and reactive mesothelium in diagnostic cytology. Both clones of anti-CD146 antibody, OJ79 and EPR3208, showed specificity of $100 \%$ for discrimination between malignant pleural mesothelioma and reactive mesothelium. Sensitivity of detection for malignant pleural mesothelioma was 94\% for OJ79 and 90\% for EPR3208. In addition, we found that OJ79 and EPR3208 were complementary in diagnosis of three malignant pleural mesothelioma cases. One OJ79-negative case (malignant pleural 

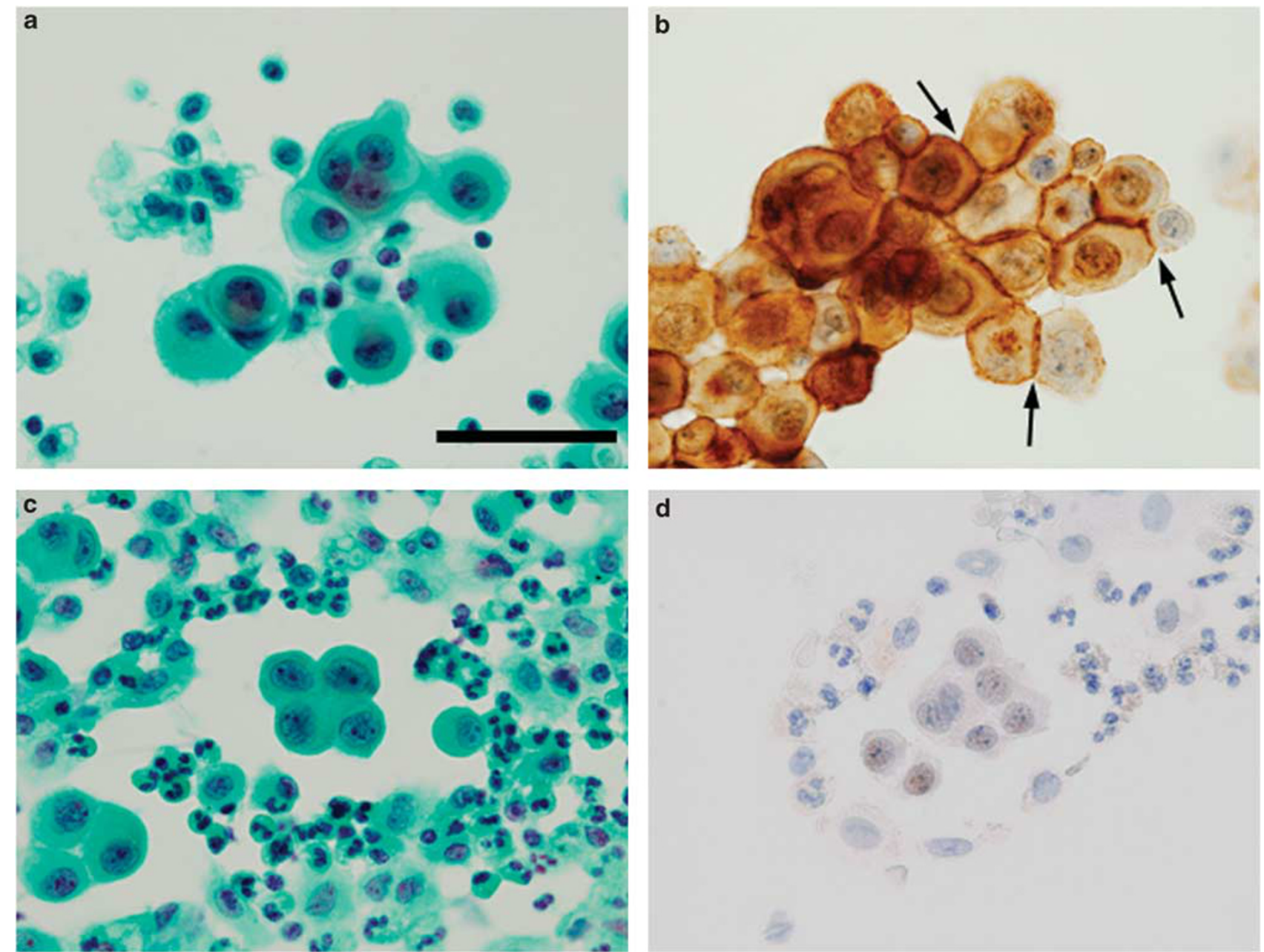

Figure 1 Expression of CD146 detected by clone OJ79 in malignant pleural mesothelioma, but not in reactive mesothelium. (a, b) Malignant pleural mesothelioma-case no. 8. (c, d) Reactive mesothelium—case no. 13. (a, c) Papanicolaou stain. (b, d) Immunostaining with OJ79. Mesothelioma cells showed membranous immunostaining with strong signals at the apposing surfaces of cell-cell interactions (arrows) (b), but reactive mesothelial cells showed no or faint expression of CD146 (d). Scale bar indicates $50 \mu \mathrm{m}$.

mesothelioma-case no.15) was positive for CD146 by EPR3208, and two EPR3208-negative cases (malignant pleural mesothelioma-case nos. 2 and 7) were CD146 positive by OJ79. These results show that CD146 is a marker capable of discriminating between malignant pleural mesothelioma and reactive mesothelium, in agreement with the recent findings by Bidlingmaier et $a l^{27}$ that CD146 has been detected in mesothelioma with high frequency but not in one case of normal mesothelium using tissue microarrays.

Comparison between CD146 and EMA found one case of malignant pleural mesothelioma with EMAnegative and CD146-positive staining (case no.12) and three cases of reactive mesothelium with EMApositive and CD146-negative staining (case nos. 8, 13, and 17). These results indicate that CD146 and EMA are almost equal as a positive marker for malignant pleural mesothelioma, but CD146 is more reliable as a negative marker for reactive mesothelium. Immunocytochemistry of CD146, together with EMA, may support the diagnostic accuracy for discrimination between malignant pleural mesothelioma and reactive mesothelium in effusion cytology.

The CD146 expression has been reported in nonsmall-cell lung cancer, and seems to be associated with shorter survival of patients with adenocarcinoma of the lung. ${ }^{28}$ In our study, reactive mesothelial cells showed no expression of CD146, but CD146 was expressed in adenocarcinoma cells that present in pleural effusions. These findings indicate that CD146 immunostaining cannot be applied to discriminate between malignant pleural mesothelioma and metastatic adenocarcinoma in the thoracic cavity.

Cytopathological features of mesothelioma cells in effusion fluids are characterized by cell-in-cell engulfment, mirror ball-like cell clusters, and multinucleated cells, which may result from adhesion and fusion of cells. Our immunocytochemical staining of effusion fluids from malignant pleural 

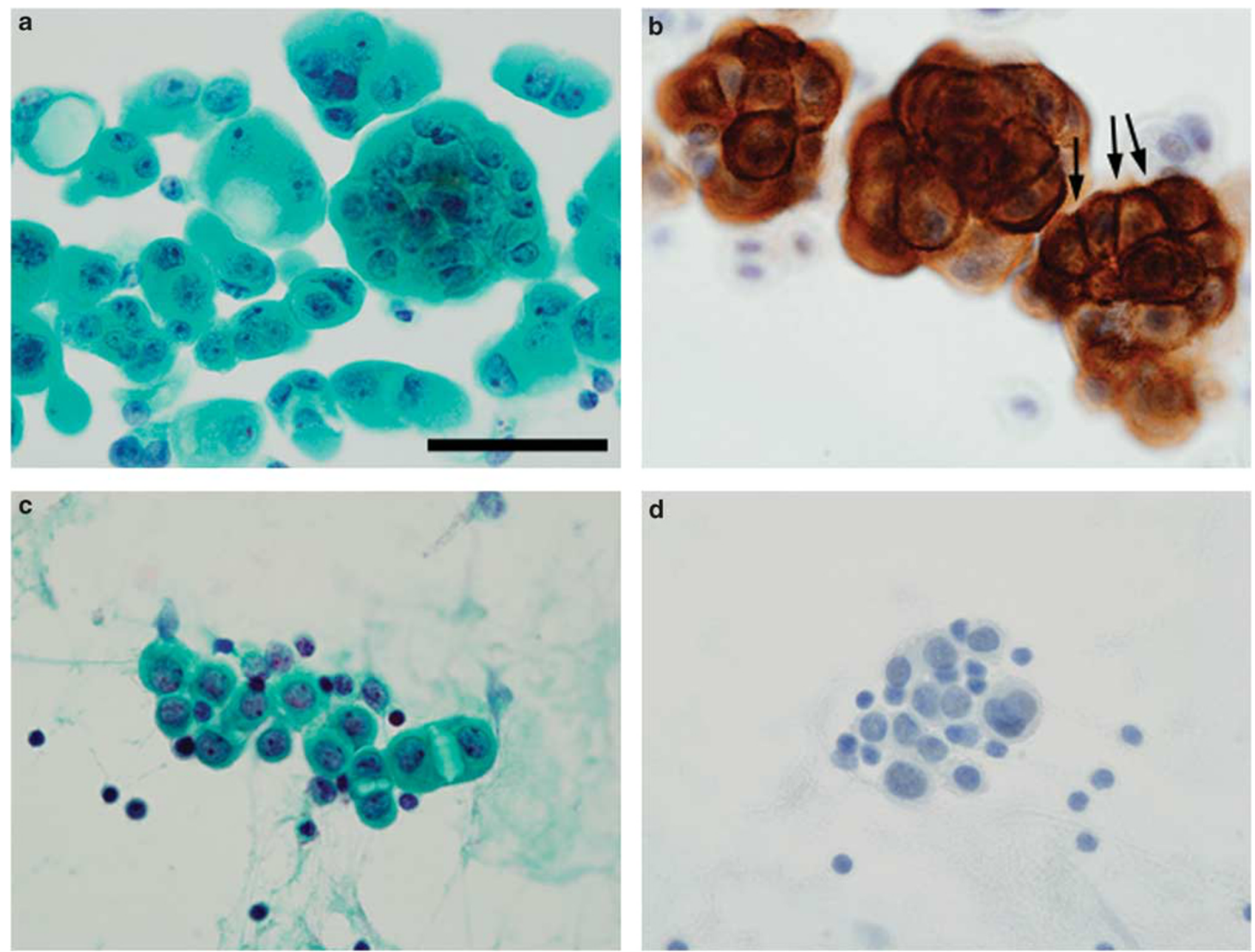

Figure 2 Expression of CD146 detected by clone EPR3208 in malignant pleural mesothelioma, but not in reactive mesothelium. (a, b) Malignant pleural mesothelioma—case no. 17. (c, d) Reactive mesothelium—case no. 10. (a, c) Papanicolaou stain. (b, d) Immunostaining with EPR3208. Mesothelioma cells showed membranous immunostaining with strong signals at the apposing surfaces of cell-cell interactions (arrows) (b), but reactive mesothelial cells showed no expression of CD146 (d). Scale bar indicates $50 \mu \mathrm{m}$.

mesothelioma patients revealed strong immunoreactivity of CD146 at the apposing surfaces of cell-cell interactions on the plasma membrane of mesothelioma cells. These findings strongly suggest involvement of cell adhesion molecules including CD146 in manifestation of characteristics of mesothelioma cells and development of malignant pleural mesothelioma.

OJ79, a clone of anti-CD146 antibody, was able to detect CD146 expression without antigen retrieval by heat treatment, and the immunodetectability of OJ79 decreased after heat treatment. It is possible that OJ79 might recognize a heat-unstable epitope. On the other hand, target antigen retrieval by heat treatment was found to result in marked increases in immunodetectability of EPR3208, another clone of anti-CD146 antibody. It is likely that OJ79 and EPR3208 recognize distinct epitopes of CD146. Preliminary experiments showed that OJ79 and EPR3208 were able to detect CD146 in formalinfixed and paraffin-embedded cell block specimens of effusion fluids. These clones might be applied to immunohistochemical analysis of formalin-fixed and paraffin-embedded surgical specimens from patients with malignant mesothelioma.

It has been reported that CD146 is expressed in most advanced melanomas, but only sporadically in benign melanocytic nevi and thin primary melanomas with low probability of metastasis. ${ }^{21,22}$ In addition, increased CD146 expression has been shown to be closely related to advanced stages of prostate and ovarian cancers. ${ }^{23-25}$ On the other hands, CD146 has been shown to act as a tumor suppressor in breast cancer, ${ }^{29}$ suggesting that the function of CD146 is different according to the type of malignancies.

Bidlingmaier et $a l^{27}$ have recently identified CD146 as the surface antigen recognized by the targeting single-chain antibody that was capable of internalizing epitopes on the plasma membrane of mesothelioma cells. Using a mesothelioma xenograft model, the single-chain antibody against CD146 was 

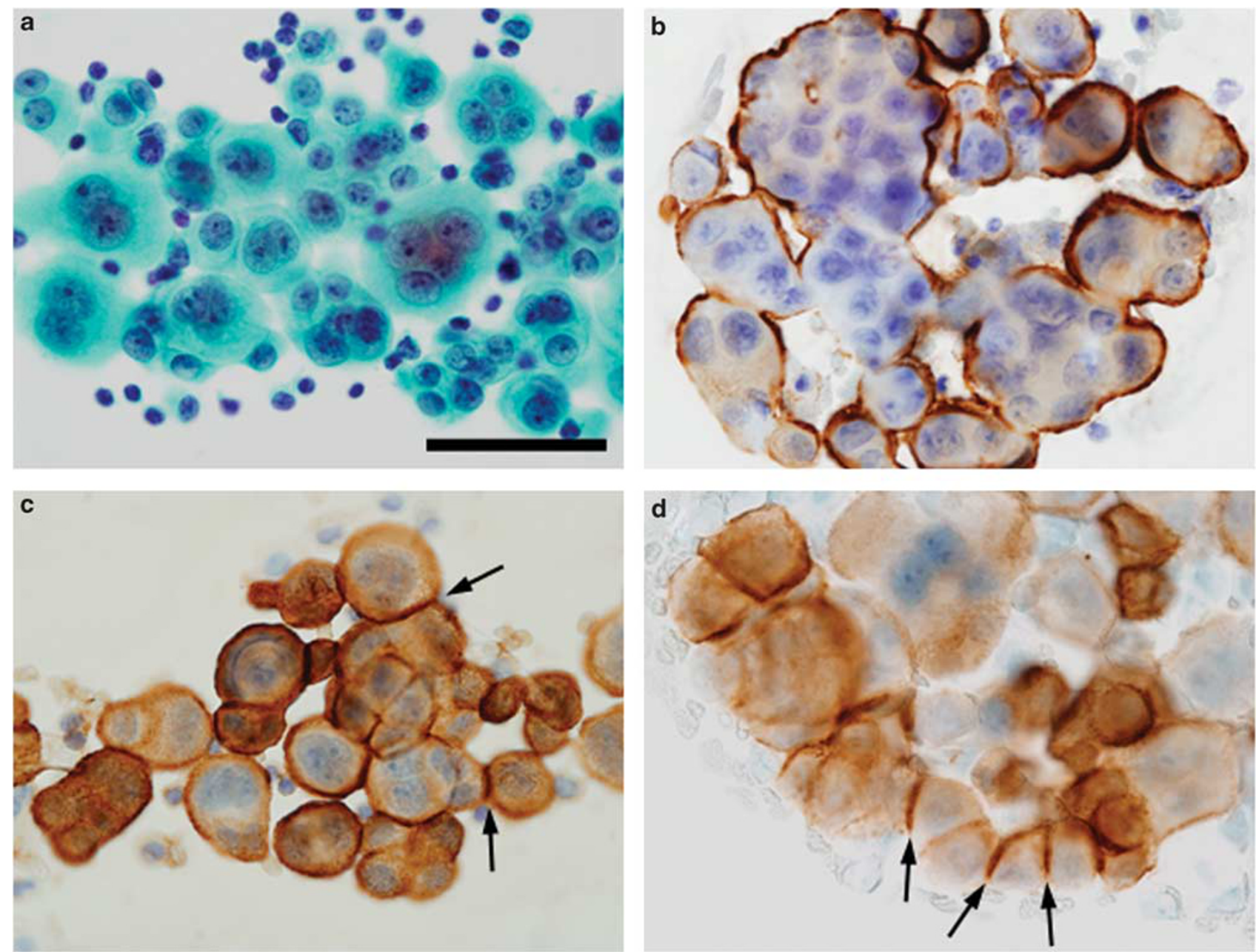

Figure 3 Different expression patterns of CD146 and EMA. (a-d) Malignant pleural mesothelioma-case no. 11. (a) Papanicolaou stain. (b- d) Immunostaining with EMA, OJ79, and EPR3208, respectively. CD146 expression is detected by OJ79 and EPR3208 at the apposing surfaces of cell-cell interactions (arrows) (c, d), whereas EMA expression is mainly detected in the periphery of cell clusters (b). Scale bar indicates $50 \mu \mathrm{m}$.
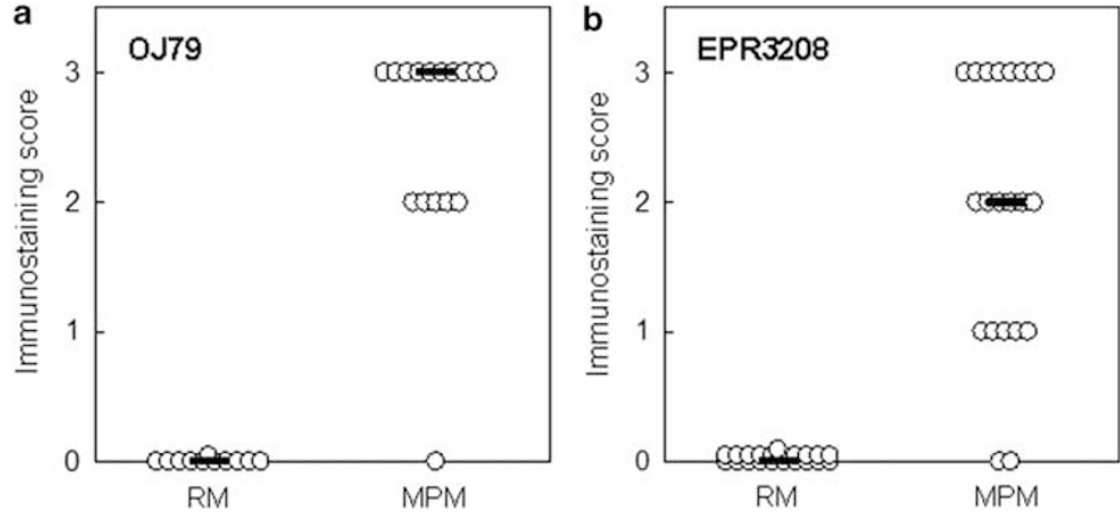

Figure 4 Distributions of CD146 immunostaining score. (a) Score of OJ79. (b) Score of EPR3208. Open circles represent immunostaining scores listed in Table 1 and the horizontal bars indicate the median value. CD146 expression detected by both clones OJ79 and EPR3208 in malignant pleural mesothelioma was significantly higher than that in reactive mesothelium $(P<0.001)$. RM, reactive mesothelium; MPM, malignant pleural mesothelioma.

shown to possess the potential for targeting therapy of mesothelioma. In addition, studies on immunotherapy using a fully human anti-CD146 antibody
(ABX-MA1) showed that ABX-MA1 inhibited growth and lung metastasis of melanoma cells injected subcutaneously into nude mice. ${ }^{30}$ As the 
present study showed that mesothelioma cells, but not reactive mesothelial cells, express CD146, antiCD146 antibody may have potential for effective treatment of malignant pleural mesothelioma.

In summary, CD146, a cell adhesion molecule associated with progression of several malignancies, was expressed in mesothelioma cells but not in reactive mesothelial cells in effusion fluids. The sensitivity of detection for malignant pleural mesothelioma was 94 and 90\% for OJ79 and EPR3208, respectively, and the specificity for discrimination between malignant pleural mesothelioma and reactive mesothelium was $100 \%$ for both clones. We propose that CD146 is a sensitive and specific immunocytochemical marker enabling differential diagnosis of malignant pleural mesothelioma from reactive mesothelium.

\section{Acknowledgements}

This work was supported in part by Grants-in-Aid for Scientific Research and Hitec Research Center Grant from the Ministry of Education, Science, Sports, Culture, and Technology of Japan, and Grants-in-Aid for Researchers, Hyogo College of Medicine. We thank Ms Michiko Kakihana and Ms Mio Ohkabe of Hyogo College of Medicine for their technical and secretarial assistance.

\section{Disclosure/conflict of interest}

The authors declare no conflict of interest.

\section{References}

1 Robinson BW, Lake RA. Advances in malignant mesothelioma. N Engl J Med 2005;353:1591-1603.

2 Tsao AS, Wistuba I, Roth JA, et al. Malignant pleural mesothelioma. J Clin Oncol 2009;27:2081-2090.

3 Robinson BWS, Chahinian AP, (eds) Mesothelioma. Martin Dunitz: London, 2002, pp 366.

4 Whitaker D. The cytology of malignant mesothelioma. Cytopathology 2000;11:139-151.

5 Stevens MW, Leong AS, Fazzalari NL, et al. Cytopathology of malignant mesothelioma: a stepwise logistic regression analysis. Diagn Cytopathol 1992;8: 333-341.

6 Churg A, Cagle PT, Roggli VL. Cytology of the serosal surfaces. In: Silverberg SG, Sobin LH (eds). AFIP Atlas of Tumor Pathology: Tumors of the Serosal Membranes. 4th series, fascicle 3. American Registry of Pathology in collaboration with the Armed Forces Institute of Pathology: Washington, DC, 2006, pp 11-31.

7 Cakir E, Demirag F, Aydin M, et al. Cytopathologic differential diagnosis of malignant mesothelioma, adenocarcinoma and reactive mesothelial cells: a logistic regression analysis. Diagn Cytopathol 2009; 37:4-10.

8 Fetsch PA, Abati A. Immunocytochemistry in effusion cytology: a contemporary review. Cancer 2001;93: 293-308.
$9 \mathrm{Pu}$ RT, Pang Y, Michael CW. Utility of WT-1, p63, MOC31, mesothelin, and cytokeratin (K903 and CK5/6) immunostains in differentiating adenocarcinoma, squamous cell carcinoma, and malignant mesothelioma in effusions. Diagn Cytopathol 2008;36:20-25.

10 Wolanski KD, Whitaker D, Shilkin KB, et al. The use of epithelial membrane antigen and silver-stained nucleolar organizer regions testing in the differential diagnosis of mesothelioma from benign reactive mesothelioses. Cancer 1998;82:583-590.

11 Hurlimann J. Desmin and neural marker expression in mesothelial cells and mesotheliomas. Hum Pathol 1994;25:753-757.

12 Kato Y, Tsuta K, Seki K, et al. Immunohistochemical detection of GLUT-1 can discriminate between reactive mesothelium and malignant mesothelioma. Mod Pathol 2007;20:215-220.

13 Ramael M, Lemmens G, Eerdekens C, et al. Immunoreactivity for p53 protein in malignant mesothelioma and non-neoplastic mesothelium. J Pathol 1992;168: 371-375.

14 Cury PM, Butcher DN, Corrin B, et al. The use of histological and immunohistochemical markers to distinguish pleural malignant mesothelioma and in situ mesothelioma from reactive mesothelial hyperplasia and reactive pleural fibrosis. J Pathol 1999;189: 251-257.

15 King J, Thatcher N, Pickering C, et al. Sensitivity and specificity of immunohistochemical antibodies used to distinguish between benign and malignant pleural disease: a systematic review of published reports. Histopathology 2006;49:561-568.

16 Shen J, Pinkus GS, Deshpande V, et al. Usefulness of EMA, GLUT-1, and XIAP for the cytologic diagnosis of malignant mesothelioma in body cavity fluids. Am J Clin Pathol 2009;131:516-523.

17 Davidson B, Nielsen S, Christensen J, et al. The role of desmin and N-cadherin in effusion cytology: a comparative study using established markers of mesothelial and epithelial cells. Am J Surg Pathol 2001;25: 1405-1412.

18 Saad RS, Cho P, Liu YL, et al. The value of epithelial membrane antigen expression in separating benign mesothelial proliferation from malignant mesothelioma: a comparative study. Diagn Cytopathol 2005;32: 156-159.

19 Edelman GM, Crossin KL. Cell adhesion molecules: implications for a molecular histology. Annu Rev Biochem 1991;60:155-190.

20 Albelda SM. Role of integrins and other cell adhesion molecules in tumor progression and metastasis. Lab Invest 1993;68:4-17.

21 Lehmann JM, Riethmüller G, Johnson JP. MUC18, a marker of tumor progression in human melanoma, shows sequence similarity to the neural cell adhesion molecules of the immunoglobulin superfamily. Proc Natl Acad Sci USA 1989;86:9891-9895.

22 Shih IM, Elder DE, Speicher D, et al. Isolation and functional characterization of the A32 melanomaassociated antigen. Cancer Res 1994;54:2514-2520.

$23 \mathrm{Wu}$ GJ, Varma VA, Wu MW, et al. Expression of a human cell adhesion molecule, MUC18, in prostate cancer cell lines and tissues. Prostate 2001;48: 305-315.

$24 \mathrm{Wu} \mathrm{GJ}$, Wu MW, Wang SW, et al. Isolation and characterization of the major form of human MUC18 cDNA gene and correlation of MUC18 over-expression 
in prostate cancer cell lines and tissues with malignant progression. Gene 2001;279:17-31.

25 Aldovini D, Demichelis F, Doglioni C, et al. M-CAM expression as marker of poor prognosis in epithelial ovarian cancer. Int J Cancer 2006;119:1920-1926.

26 Kimura N, Dota K, Araya Y, et al. Scoring system for differential diagnosis of malignant mesothelioma and reactive mesothelial cells on cytology specimens. Diagn Cytopathol 2009;37:885-890.

27 Bidlingmaier S, He J, Wang Y, et al. Identification of MCAM/CD146 as the target antigen of a human monoclonal antibody that recognizes both epithelioid and sarcomatoid types of mesothelioma. Cancer Res 2009;69:1570-1577.

28 Kristiansen G, Yu Y, Schlüns K, et al. Expression of the cell adhesion molecule CD146/MCAM in non-small cell lung cancer. Anal Cell Pathol 2003;25:77-81.

29 Shih LM, Hsu MY, Palazzo JP, et al. The cell-cell adhesion receptor Mel-CAM acts as a tumor suppressor in breast carcinoma. Am J Pathol 1997;151:745-751.

30 Mills L, Tellez C, Huang S, et al. Fully human antibodies to MCAM/MUC18 inhibit tumor growth and metastasis of human melanoma. Cancer Res 2002;62:5106-5114. 